\title{
BMJ open Clinician beliefs and attitudes about home haemodialysis: a multinational interview study
}

\author{
Allison Tong, ${ }^{1,2}$ Suetonia Palmer, ${ }^{3}$ Braden Manns, ${ }^{4}$ Jonathan C Craig, ${ }^{1}$ \\ Marinella Ruospo, ${ }^{5}$ Letizia Gargano, ${ }^{5}$ David W Johnson, ${ }^{6}$ Jörgen Hegbrant, ${ }^{7}$ \\ Måns Olsson, ${ }^{8}$ Steven Fishbane, ${ }^{9}$ Giovanni F M Strippoli ${ }^{2,10,11,12}$
}

To cite: Tong A, Palmer S, Manns B, et al. Clinician beliefs and attitudes about home haemodialysis: a multinational interview study. BMJ Open 2012;2: e002146. doi:10.1136/ bmjopen-2012-002146

- Prepublication history for this paper are available online. To view these files please visit the journal online (http://dx.doi.org/10.1136/ bmjopen-2012-002146).

Received 21 September 2012 Revised 4 November 2012 Accepted 13 November 2012

This final article is available for use under the terms of the Creative Commons Attribution Non-Commercial 2.0 Licence; see http://bmjopen.bmj.com

For numbered affiliations see end of article.

\section{Correspondence to} Professor Giovanni Strippoli; gfmstrippoli@gmail.com

\section{ABSTRACT}

Objectives: To explore clinician beliefs and attitudes about home haemodialysis in global regions where the prevalence of home haemodialysis is low, and to identify barriers to developing home haemodialysis services and possible strategies to increase acceptance and uptake of home haemodialysis.

Design: Semistructured interviews, thematic analysis.

Setting: 15 dialysis centres in Italy, Portugal, France, Germany, Sweden and Argentina.

Participants: 28 nephrologists and 14 nurses caring for patients receiving in-centre haemodialysis.

Results: We identified four major themes as being central to clinician beliefs about home haemodialysis in regions without established services: external structural barriers (ready access to dialysis centres, inadequate housing conditions, unstable economic environment); dialysis centre characteristics (availability of alternative treatments, competing service priorities, commercial interests); clinician responsibility and motivation (preserving safety and security, lack of awareness, knowledge and experience, potential to offer lifestyle benefits, professional interest and advancement); and cultural apprehension (an unrelenting imposition, carer burden, attachment to professional healthcare provision, limited awareness).

Conclusions: Despite recognising the potential benefits of home haemodialysis, clinicians practicing in Europe and South America felt apprehensive and doubted the feasibility of home haemodialysis programmes. Programmes that provide clinicians with direct experience of home haemodialysis could increase acceptance and motivation for home-based haemodialysis, as might service prioritisation and funding models that favour home haemodialysis.

\section{INTRODUCTION}

Approximately 2 million people worldwide receive chronic dialysis treatment for end-stage kidney disease with annual direct healthcare costs that exceed USD 60 billion. ${ }^{1}$ The global dialysis population is expanding rapidly in the context of constrained workforce and financial

\section{ARTICLE SUMMARY}

Article focus

- To elicit clinician beliefs and attitudes about home haemodialysis in global regions where the prevalence of home haemodialysis is low.

- To identify barriers to developing home haemodialysis services and possible strategies to increase the acceptance and uptake of home haemodialysis.

Key messages

- Nephrologists and dialysis nurses in regions where home haemodialysis is not established recognise the potential benefits but are pessimistic about the feasibility of developing such programmes.

- Direct clinician experience and education as well as improved reimbursement for home haemodialysis may assist the establishment of home haemodialysis services.

- Clinicians express concern about the quality and safety of home haemodialysis, which may be addressed by increased experience of home dialysis care.

Strengths and limitations of this study

- We actively sought a broad range of views and experiences through purposive sampling.

- Our study included only clinicians within a large dialysis service provider in Europe and South America.

resources. In the last decade the haemodialysis populations in China, India and Brazil increased $15 \%$ each year ${ }^{2}$ and in Australia the dialysis population nearly doubled. ${ }^{3}$ People who require dialysis treatment experience severely impaired quality of life and role function and have a markedly increased risk of mortality compared with the general population. Approximately $10-20 \%$ of people on in-centre haemodialysis die each year and only about $10 \%$ are employed. ${ }^{4}$ In contrast to in-centre dialysis, people on home haemodialysis may 
experience a life-expectancy approaching that of a deceased donor kidney transplant recipient. ${ }^{5}$ When analyses are controlled for comorbidity, home haemodialysis is associated with better outcomes than in-centre haemodialysis for most measures of effectiveness. ${ }^{5}{ }^{6}$

Although home haemodialysis is consistently associated with markedly improved quality of life and patient rehabilitation, the prevalence of home haemodialysis as a proportion of all dialysis care varies nearly 100 -fold globally. Home haemodialysis is common in New Zealand and Australia (20\% of all dialysis patients) but is rare or unavailable in parts of Europe and Scandinavia, the USA and South America. ${ }^{7}$ The reasons for the wide variation in uptake are unclear, although higher home haemodialysis use is characterised by local advocacy and experience combined with service centralisation and favourable reimbursement. ${ }^{8} 9$ When patients are educated about home dialysis therapies, about one-half choose self-care dialysis ${ }^{10}$ and National Institute for Clinical Excellence guidelines recommend that all suitable patients should be offered the choice between home haemodialysis and in-centre haemodialysis in a hospital or satellite clinic. ${ }^{11}$

Clinicians are critical to the effective implementation of home haemodialysis services ${ }^{12}{ }^{13}$ to improve patient choice and facilitate increased dialysis service capacity in response to expanding patient populations. ${ }^{14}$ To date, there is little published research describing the attitudes of clinicians towards home haemodialysis in areas where home haemodialysis is rare or non-existent. In view of the absence of home haemodialysis programmes in some areas within Europe, Scandinavia and South America, the aim of this study was to explore the perceptions of home haemodialysis from nephrologists and dialysis nurses in these regions.

\section{METHODS}

We invited practising nephrologists and dialysis nurses from Europe and South America (France, Germany, Italy, Portugal, Sweden and Argentina) involved in the care of patients on chronic haemodialysis in facilities that do not currently provide home haemodialysis services. We used purposive sampling to select both male and female clinicians from several geographical areas in Europe and South America and with diverse clinical experience of haemodialysis to ensure a broad range of views and experiences of haemodialysis provision. All participants were recruited from centres within Diaverum, a large dialysis service provider. Details of dialysis services provision and reimbursement in the participating countries are provided in table 1 . In all contributing countries, access to dialysis services is universal, but the percentage of private providers and reimbursement models varies to a great extent. Ethics approval was provided by all participating centres.

To elicit clinician beliefs and experiences, we developed a semi-structured interview guide based on a literature review on home haemodialysis (any haemodialysis at home including thrice weekly dialysis or frequent haemodialysis performed during the day or night) ${ }^{15-24}$ and discussions between the research team (box 1). The interviews focused on knowledge of home haemodialysis, perceived benefits and risks, potential facilitators and barriers to home haemodialysis, training and support needs of clinicians, and clinical attitudes towards home haemodialysis. One author (AT) conducted one face-toface semistructured qualitative interview with each participant in their office or hospital meeting room. An interpreter was present during interviews with non-English-speaking participants $(n=20)$. We ceased recruitment when theoretical saturation was reached. We recorded contextual details after each interview. We digitally audio-recorded and transcribed verbatim all interviews in full.

We entered transcripts into a software package for storing, coding and searching qualitative data (HyperRESEARCH V.3.0.2., ResearchWare, Inc, 2011, http://www.research.com). Drawing on grounded theory and thematic analysis, ${ }^{25} 26$ one author (AT) coded the transcripts and recorded concepts inductively,

Table 1 Characteristics of in-centre haemodialysis service provision and reimbursement in participating countries

\begin{tabular}{|c|c|c|c|c|c|c|c|c|c|c|}
\hline \multirow[b]{2}{*}{ Country } & \multicolumn{3}{|c|}{ Type of providers (\%) } & \multirow[b]{2}{*}{$\begin{array}{l}\mathrm{PD} \\
\text { rate }\end{array}$} & \multicolumn{6}{|c|}{ In-clinic haemodialysis reimbursement coverage for private providers } \\
\hline & Public & $\begin{array}{l}\text { Private } \\
\text { for } \\
\text { profit }\end{array}$ & $\begin{array}{l}\text { Private } \\
\text { non-profit }\end{array}$ & & $\begin{array}{l}\text { Basic } \\
\text { service* }^{*}\end{array}$ & Nephrologist & $\begin{array}{l}\text { Laboratory } \\
\text { tests }\end{array}$ & Erythropoietin & $\begin{array}{l}\text { Vascular } \\
\text { access }\end{array}$ & Transport \\
\hline France & 28 & 35 & 37 & 8 & $\mathrm{X}$ & & & & & \\
\hline Portugal & 15 & 85 & - & 7 & $\mathrm{X}$ & $\mathrm{X}$ & $\mathrm{X}$ & $\mathrm{X}$ & $\mathrm{X}$ & $\mathrm{X}$ \\
\hline Italy & 75 & 25 & - & 11 & $x$ & $x$ & $x$ & & & \\
\hline Germany & $10 \dagger$ & 52 & 38 & 5 & $x$ & $x$ & $x$ & & & \\
\hline Sweden & 90 & 10 & - & 22 & $x$ & $X$ & $x$ & $\ddagger$ & & \\
\hline Argentina & 5 & 95 & - & 5 & $x$ & $X$ & $x$ & $x$ & $X$ & $X$ \\
\hline
\end{tabular}


Box 1 Semistructured interview questions and topic guide

- Brief explanation of the study and review of duration of involvement in haemodialysis-related care (to introduce and provide context)

Ask about:

- General clinician experiences of providing dialysis care: Which are the most important aspects of caring for a person on haemodialysis, which are the most difficult challenges encountered in caring for patients on dialysis, describe key patient problems with haemodialysis and if the clinician was in charge of their haemodialysis unit, what would they change to improve experiences of patients and/or staff

- Knowledge of home haemodialysis: what has he or she heard about home haemodialysis and is there anything else he or she would like to know about?

- Perceived benefits: Are there any benefits or advantages of home haemodialysis compared with in-centre haemodialysis (freedom, flexibility, convenience, simplicity, effectiveness, dietary freedom, waiting at hospital for dialysis, less travel time, ability to work or socialize, more family involvement and more privacy)?

- Perceived risks: What does the clinician see are the risks of patients doing haemodialysis at home? (isolation, risk/safety, family burden and home modification)?

- Facilitators: What sorts of programmes or support does the clinician think would help patients consider doing home haemodialysis? (plumbing, support, group meetings, emergency contact and clinician support)

- Barriers: What does the clinician see are the barriers to home haemodialysis and how could these be addressed?

- Role: If home haemodialysis was offered, what does the clinician think his or her role would be? How does the clinician think his or her current roles and responsibilities will change if home haemodialysis was offered by the clinic?

- Training and support: What additional support or training would the clinician require before home haemodialysis could be offered in his or her practice?

- Attitudes: Would the clinician be supportive of offering home haemodialysis to patients in his or her clinic? Why? Does the clinician think his or her colleagues would be supportive, and why?

grouped similar concepts and identified patterns and relationships in the data. AT discussed the preliminary coding scheme with MR who had independently reviewed the transcripts. The coding structure was refined until we captured all concepts relating to the perspectives of clinician beliefs and attitudes about home haemodialysis. To enhance the analytical framework and potential for offering meaningful insight, interpretation of the data and preliminary themes was discussed by the research team.

\section{RESULTS}

We interviewed 42 of 43 invited clinicians (98\%) in October and November 2011. One could not participate because of clinical commitments. Each interview lasted between 25 and $50 \mathrm{~min}$. Most participants were nephrologists $(n=28)$, were from Portugal $(n=16)$ or France $(n=13)$ and were men $(n=29)$ (table 2$)$. The clinicians had between 4 and 28 years of nephrology experience and 10 had previous experience in home haemodialysis.

In our analytical framework we identified four themes that were central to clinician beliefs and attitudes towards home haemodialysis: structural barriers (ready access to existing treatment, inadequate housing and unstable economic environment), dialysis centre characteristics (alternative treatment options, competing service priorities and commercial interests), clinician responsibility and motivation (safety and security, knowledge and awareness, lifestyle benefits and professional development) and cultural apprehension (unrelenting imposition, carer burden, attachment to professionals and limited awareness) (figure 1). Table 3 provides a selection of quotes from the clinicians to illustrate each theme.

\section{STRUCTURAL BARRIERS}

The theme of structural barriers encompassed views on the physical and financial barriers to home haemodialysis that were believed reduce the motivation and capacity of providers to offer home haemodialysis programmes. Access to dialysis centres related to the close proximity of most patients to existing dialysis services that clinicians thought reduced the need for home-based care. Housing conditions emphasised the concerns of clinicians that accommodation and utilities were frequently inadequate to support home haemodialysis. The economic environment described the widespread anxiety felt by clinicians that dialysis providers and patients

Figure 1 Thematic schema.

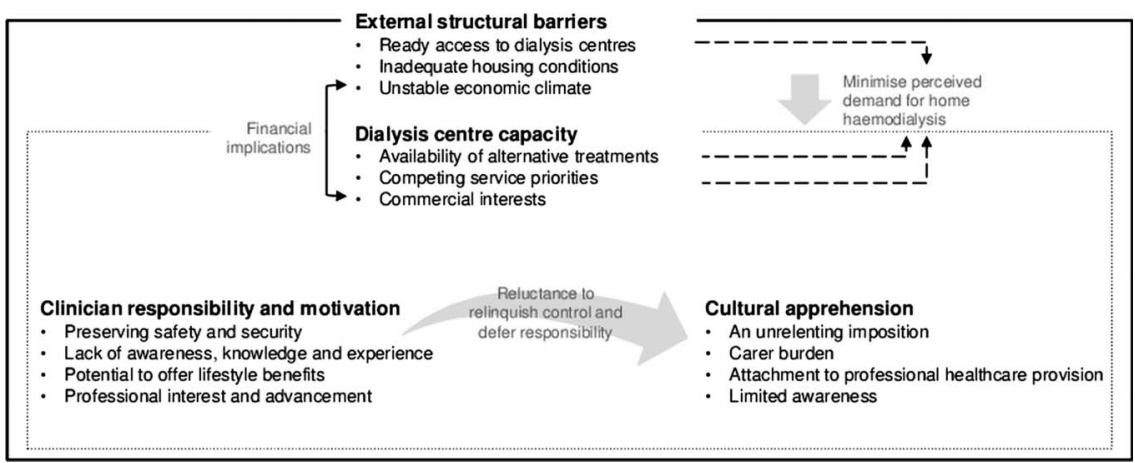




\begin{tabular}{|c|c|c|}
\hline Characteristics & Interviewees (n) & (\%) \\
\hline \multicolumn{3}{|l|}{ Gender } \\
\hline Male & 29 & (69) \\
\hline Female & 13 & (31) \\
\hline \multicolumn{3}{|l|}{ Age } \\
\hline $20-29$ & 1 & (2) \\
\hline $30-39$ & 6 & (14) \\
\hline $40-49$ & 17 & (41) \\
\hline $50-59$ & 13 & (31) \\
\hline $60-69$ & 5 & (12) \\
\hline \multicolumn{3}{|l|}{ Role } \\
\hline Nephrologist & 28 & (67) \\
\hline Nurse & 14 & (33) \\
\hline \multicolumn{3}{|c|}{ Years of nephrology experience } \\
\hline$\leq 10$ & 6 & (14) \\
\hline $11-20$ & 17 & (41) \\
\hline $21-30$ & 10 & (24) \\
\hline$>30$ & 9 & (21) \\
\hline \multicolumn{3}{|c|}{ Location (city, country) of primary dialysis centre } \\
\hline Marseille, France & 12 & (29) \\
\hline Arles, France & 1 & (2) \\
\hline Bari, Italy & 1 & (2) \\
\hline Rome, Italy & 1 & (2) \\
\hline Potsdam, Germany & 1 & (2) \\
\hline Buenos Aires, Argentina & 1 & (2) \\
\hline Taranto, Italy & 1 & (2) \\
\hline Stockholm, Sweden & 1 & (2) \\
\hline Malmo, Sweden & 1 & (2) \\
\hline Marsala, Italy & 3 & (7) \\
\hline Nissoria, Italy & 3 & $(7)$ \\
\hline Lisbon, Portugal & 16 & (38) \\
\hline
\end{tabular}

could not afford new home haemodialysis initiatives, particularly in the current financial climate.

\section{Ready access to dialysis centres}

Almost all clinicians perceived that ready access to existing dialysis clinics and patient transport reimbursement reduced the need for home haemodialysis and, because of this, acquisition of capital equipment for home haemodialysis might be unjustified.

\section{Inadequate housing conditions}

Clinicians considered that home haemodialysis was impossible for patients with small houses or inadequate hygiene. However, when prompted, many participants acknowledged they were not familiar with the smaller size of haemodialysis machines designed for home use. Clinicians mentioned that some patients could not access clean water or did not own their homes and were therefore were not permitted to make necessary plumbing or electrical modifications.

\section{Unstable economic environment}

Clinicians believed that the feasibility of establishing home haemodialysis programmes is determined by dedicated government funding which was unlikely in the present economic climate. Most clinicians were employed by dialysis providers in European countries in financial crisis which, together with government health budget cuts, were perceived as significant barriers to developing home haemodialysis services, particularly as they felt the financial risks of such programmes were uncertain.

\section{DIALYSIS CENTRE CHARACTERISTICS}

The theme centre capacity described the features of dialysis centre practice that quell demand for home haemodialysis. Alternative treatment options referred to clinic centres offering home-based peritoneal dialysis and self-care haemodialysis services in preference to home haemodialysis to offer patients more flexibility and autonomy. Competing priorities related to the need for dialysis centres to focus on specific dialysis programmes often at the expense of home haemodialysis initiatives because of limited resources and reimbursement policies. Commercial interests described clinician anxiety over the lack of centralised reimbursement for home haemodialysis; most felt that increased uptake of home haemodialysis would reduce dialysis provider income and, as a consequence, impact on clinician job security.

\section{Availability of alternative treatments}

Clinicians believed that alternative programmes offered by dialysis clinics weakened the demand for home haemodialysis programmes. Such treatment options, which improve patient autonomy at lower cost, include in-centre self or limited care haemodialysis, in-centre nocturnal haemodialysis and home peritoneal dialysis.

\section{Competing service priorities}

All clinicians believed that dialysis centres should deliver high-quality care and endeavour to maximise patient satisfaction. Some felt that their centre had insufficient staff and centre facilities to support home haemodialysis training and sustain home haemodialysis programmes. In addition, some centres were focused on establishing and maintaining other newer treatment programmes including in-centre nocturnal haemodialysis as well as home peritoneal dialysis and, accordingly, could not diversify further resources into home haemodialysis programmes. Some clinicians suggested that centres which had reached maximum capacity could consider offering home haemodialysis.

\section{Commercial interests}

Some clinicians acknowledged that dialysis in their regions is an 'industry.' They speculated that centres would incur an overall financial loss if home haemodialysis was offered. Some were anxious that their centre would lose patients and income if home haemodialysis was offered, and staff might be made redundant as a result. Clinicians believed dialysis providers would not invest in new infrastructure for home haemodialysis 
Table 3 Quotations from clinicians to illustrate each theme

\section{Themes Quotations from participants in study}

Structural barriers

Ready access The transport is paid back by social security. It's easier for them to come to the centre. (Male nephrologist, to in-centre 40s, France)

dialysis

We have an in clinic environment more or less every 10 kilometres in capital cities and every 30 kilometres in rural areas... why should you buy additional equipment to comfort people to get treatment at home? ... there is just simply no need to do it at home ... (Female nephrologist, 40s, Germany)

Inadequate We have some patients with very precarious, fragile homes, shaky homes made of wood, not strong, and housing not hygienic. (Male nurse, 40s, Portugal)

I think it could be a problem for them to manage themselves if they have little space in their house to put the dialysis machine and especially if they are not the owner of their house ... maybe the costs of the electricity or hydraulics. (Male nephrologist, 40s, Italy)

It's very difficult to implement when you have limitations. Because you need the thing that ... a lot of regions in Argentina don't have ... good water. (Male nephrologist, 60s, Argentina)

Unstable For home haemodialysis in countries like Argentina, Chile, Uruguay, you have financial and economic economic limits. It's very expensive. The very big cost is around the machine, the dialysis machine and the water environment treatment. ... In Argentina ... and Brazil we re-use ... the haemodialysis filters. In Chile and Uruguay and Brazil, they re-use ... the blood line. (Male nephrologist, 60s, Argentina)

In my opinion today it's not a good [time] ... because of the problem with the financial economic crisis. I don't know if it is more expensive but I would think so. (Male nephrologist, 40s, Portugal)

Centre characteristics

Alternative At that time we had 15 patients on limited care, and among those, most of them did the treatment treatment options themselves. Two of them wanted to go home ... that says something ... 3 wanted to stay and didn't want the machine at home. (Male nephrologist, 50s, Sweden)

They started the home haemodialysis project and moved to the assisted, limited person assistance dialysis ... some of them were completely autonomous, independent and they did dialysis by themselves ... (Male nephrologist, 60s, Italy)

Some patients don't want to waste time during the day so they go to the centre, after dinner, they start dialysis. Most of the time they do it themselves, and they have a nurse and a doctor nearby, and they will wake at 7 or 8 o'clock, they take a bath and they go. (Male nephrologist, 60s, Portugal)

I think the other alternative maybe is peritoneal dialysis, which is in my opinion a better home dialysis. For the patients, it's much...easier. You can be at home by yourself doing it. I don't know if you can be at home by yourself doing home haemodialysis. (Male nephrologist, 40s, Portugal)

Competing They prefer to come, to the centres in Portugal, we have comfortable, good centres. (Male nephrologist, priorities $\quad 60 \mathrm{~s}$, Portugal)

We had a priority to set up extended dialysis with a nightshift dialysis... because...the patients wanted that. So that's what we've basically done the last two months and that means also that half of our clinics are not - I mean right now they're more recruiting for nocturnal dialysis rather than offering them additional options with a home haemodialysis. So we had two competing programs if you may say for younger patients. One was nocturnal dialysis and one was a peritoneal dialysis program where we doubled the numbers of our patients since January. (Female nephrologist, 40s, Germany)

You need to have also good logistics and structure for [home haemodialysis] ... you cannot just have one patient. It's very difficult to have something for very few patients so you need to have a centre or something in the region. (Male nephrologist, 50s, Sweden)

Commercial I think in terms of economics it's also better because it's a little cheaper at the beginning with no staff, interests no transportation. We can also save money without the staff. (Female nephrologist, 50s, Portugal) If we send them to home dialysis, we miss [lose] patients from our centres. It's not the most important but it's important. Because there is an economic crisis, I don't want to see my friends, my colleagues have his contract terminated because of this [home haemodialysis] project. (Male nurse, 30s, France)

If the patient goes to home dialysis the doctor might earn less money so if he does earn more with patients in the heavy [large] centres, the doctor might tell him to go in a heavy [large] centre more than home dialysis. (Male nephrologist, 40s, France)

...perhaps, also because there is no incentive for personnel and for the organization to permit the diffusion of this type of [home haemo] dialysis $\mathrm{K}$ (Male nephrologist, 40s, Italy)

So when there are no financial incentives to do home haemodialysis then people don't do it. So, people are not interested in spending $€ 15,000$ [on] the patient to set up the infrastructure...that is then only used by one patient. (Female nephrologist, 40s, Germany)

For example, the biggest barrier to peritoneal dialysis is financial. Many doctors don't put patients on peritoneal dialysis because they think they can make money with haemo [dialysis]. (Male nephrologist, 50s, Sweden) 
Table 3 Continued

\section{Themes Quotations from participants in study}

\section{Clinician responsibility and motivation}

Safety and For example, once he went to emergency unit and his wife never realised he was losing weight. He was security seven kilograms overloaded, so he had 7 kilograms of water in his body and she never noticed that. So he could just have died because of this lack of follow up or whatever. (Female nurse, 30s, France)

I don't think that security of dialysis at home is the same as in the centres. In the centres we've got a lot of protocols... They are all alone. (Male nephrologist, 30s, France)

I think it's not a good idea because today, there is an infection risk. They are not professionals at home... (Female nurse, 20s, France)

There is no benefit to leave the patient at home and it is too risky to, too many risks. We have experience with peritoneal dialysis, [patients] who dialyse at home... all come back, with peritonitis, with infections. (Female nurse, 20s, France)

There is not [an] immediate link with the doctor or a nurse if you suddenly have a problem. (Male nephrologist, 50s, Italy)

Knowledge l've been working here for 18 years and l've never really heard about that so I think it would be quite hard and to start home dialysis for patients, here it's not really policy. (Male nurse, 40s, France)

awareness So far there is no promotion, nobody knows about this so it would be a strategy to start in a good way and to put some new patients to home instead of in centre haemodialysis. (Male nephrologist, 60s, Italy) I would be really interested in hearing about the other countries and knowing really how it goes there... (Female nurse, 30s, France)

Lifestyle It's a liberty for them to be treated at any time of the day, during the night, they can work and they can have benefits other, family life, as normal as possible, that's the main benefit. He stays at home he doesn't have to travel, he can keep his job, continue, does dialysis when he has the time to do it so it can be in front of the television when he has food or whenever. (Male nephrologist, 50s, France) Home dialysis, it's a beautiful thing because you could dialyse when you want, during the night during the day, you are home and you have no problem, you are at your house, that's the beautiful thing, after you've finished, you could eat, you don't travel [to the centre], it's a beautiful thing. (Male nurse, 50s) They will feel better ... on long home haemodialysis... that is what I'm expecting. They will feel better; they will take less medication and so on, so they will be [healthier] ... (Male nephrologist, 50s, Portugal)

Professional I like new experiences. I think it is good for professional growth. (Female nephrologist, 50s, Italy) development I think it will be better for the patient and as a nurse I can do my work, my job better. (Male nurse, 40s, Portugal) Cultural apprehension

Unrelenting There is one room in the house where there is the disease .... We create more problems in the relationship imposition with the partner, who is in charge of dialysis, and sometimes the relationship becomes difficult and aggressive because of this and sometimes people can just divorce because of the dialysis problem. (Male nephrologist, 50s, France)

He didn't want to take the hospital home, doing home dialysis, he wanted the disease to stay at the hospital and home was home, no disease at home. (Male nurse, 40s, France)

The patient just wants to come and to have the treatment and to go home and don't think about. Some of them don't want to think to have the machine in the home. You are with your disease all the time because you have that at home, and they say sometimes they want just to have the treatment and after... OK, I forget about it and I have my life like everybody else. (Female nurse, 30s, France)

Carer burden They [family carers] can't be imprisoned because the father needs a person by his side in dialysis. (Female nephrologist, 40s, Portugal)

If there [are] any problems and emergencies it's quite hard here for a nurse to take care of this, so at home, if there is no experience and the person is anxious and it's their relative it would be even harder. Because the patient could be the child the wife the husband it could be harder for them to deal with it. (Male nurse, 30s, France)

Attachment to People are scared about these things, medical things that look more complicated. 'I am a normal person professionals I can't do this, I'm not able.' So they are very resistant ... (Male nephrologist, 60s, Italy) I mean if you today go to the hair cutter, you want a certain level of care right...and so we are surrounded by the experiences that whenever we get a service ... we're well taken care of. (Female nephrologist, 40s, Germany)

The Portuguese don't like the responsibilities. I think the majority of patients want others to care [for them]. (Female nephrologist, 40s, Portugal)

Traditionally, everybody is linked to the doctors, everything connected with health is linked to the doctor so it's difficult for the mind set to think about home [haemodialysis], being independent from the doctor. This is a tradition. It's always a problem of mind set; it's stronger in the south [of Italy]. (Male nephrologist, 60s, Italy) 
Table 3 Continued

\begin{tabular}{ll}
\hline Themes & Quotations from participants in study \\
& The patients that are very afraid of everything in the dialysis room, when an alarm of the machine calls, \\
they get very, very scared so I think that at home they will be very scared, because they would [not] feel \\
safe ... (Female nephrologist, 40s, Italy) \\
Our people are not so educated in health. They are very afraid of taking care of their own disease. (Female \\
nephrologist, 50s, Italy) \\
The low socio-economic level of the majority of patients-it's very difficult for them. Many people here don't \\
know how to read... and it's very difficult for them to make the treatment. But I think the principal reason is \\
they don't want to be responsible. (Female nephrologist, 40s, Portugal) \\
Maybe because of what we tell the patient when he first arrives here so we explain him all the possibilities \\
but... might not talk about home dialysis enough. We don't give enough choice to the patient K (Female \\
nurse, 40s, France) \\
We're obliged to offer them three modalities like haemodialysis, peritoneal dialysis and pre-emptive \\
transplantation... and home haemodialysis ... is not promoted in the sense that we go actively and say, you \\
know, you could also have home haemodialysis. If you feel that the patient is interested then someone \\
would maybe offer it, but we're not promoting it actively. (Female nephrologist, 40 s, Germany) \\
I think nephrologists don't talk about it to the patients in most cases. Many patients don't know that it is a \\
possibility. (Female nephrologist, 40s, Portugal) \\
There isn't educational program for these patients; there is no promotion of home haemodialysis for \\
patients. (Male nephrologist, 50s, Italy)
\end{tabular}

programmes if the capital and service-related expenses were not reimbursed by government at the levels currently provided for in-centre haemodialysis. Clinicians believed that centres needed to be sufficiently incentivised by health systems to provide home haemodialysis at rates comparable with in-centre haemodialysis before home haemodialysis could be commenced in their centres.

\section{CLINICIAN RESPONSIBILITY AND MOTIVATION}

The theme clinician responsibility and motivation described the ways in which the clinicians view their roles in dialysis care. Participants believed that home haemodialysis is beneficial for patients, but while they are interested in the idea, they are reluctant to relinquish control and defer responsibilities of medical care to patients and their carers. Preserving quality and safety referred to the need by clinicians to be directly involved in dialysis processes to ensure their patients were safe on treatment. Lack of awareness, knowledge and experience reflected the clinician's own anxieties that they are less knowledgeable in home haemodialysis but retain a desire to learn more about home haemodialysis. Potential to offer lifestyle benefits encompassed the understanding of clinicians that home haemodialysis provides patients with considerable autonomy and self-reliance that can improve their own functioning and quality of life. Professional interest and advancement described the interest clinicians have in learning more about home haemodialysis to increase their own professional skills.

\section{Preserving safety and security}

Clinicians frequently identified quality and safety as key healthcare priorities within their practice and anticipated that home haemodialysis would diminish their capacity to protect the well-being of their patients. They voiced concern that patient monitoring would be less with home haemodialysis and that patients may not adhere to their prescribed regimens. Also, they believed patients and carers would not safely manage complications such as infections or cardiovascular events. Dialysis was perceived to require medical and technical expertise. Clinicians believed that most patients were too old, dependent, sick or did not have a suitable carer to have haemodialysis safely at home. Respondents felt that some patients were unable to maintain proper hygiene standards, or understand the technical requirements of haemodialysis due to low socioeconomic and education levels.

\section{Lack of awareness, knowledge and experience}

Many clinicians acknowledged that they lacked sufficient knowledge and awareness about home haemodialysis to be confident about it as a treatment option and suggested they needed more information. For example, they suggested that learning about the experiences of home haemodialysis from colleagues and patients in other regions with established programmes would increase the potential for development of home haemodialysis services in their own centres.

\section{Potential to offer lifestyle benefits}

Clinicians identified numerous benefits of home haemodialysis, which they perceived were predominantly related to patient empowerment and lifestyle, including autonomy, flexibility, comfort and privacy, ability to work, freedom and increased time. Many believed that home haemodialysis was good particularly for young 
employed patients and a few suggested that longer dialysis sessions would improve clinical parameters and reduce the need for medications.

\section{Professional interest and advancement}

Some participants, both nephrologists and nurses, were interested and enthusiastic about offering home haemodialysis, particularly if they believed it would benefit patients. For some, home haemodialysis is a 'new' technique which would enable them to acquire additional professional skills and experiences and be better clinicians.

\section{CULTURAL APPREHENSION}

The theme cultural apprehension described the concerns that clinicians have about the disruption to families caused by home haemodialysis and burden of medical responsibility placed on unwilling patients and their carers by home-based care. The clinicians emphasised that most patients have close family networks and would be unwilling to encumber family members with the 'burden' of dialysis. They believed that patients highly value and depend on professional medical care within a clinic setting, and if severed, this might cause distress. Unrelenting imposition encompassed the clinicians' beliefs that patients would prefer to keep dialysis away from the home environment and their family and friends. Carer burden reflected the concern that clinicians hold for the ability of carers to cope with the longterm responsibility for home haemodialysis. Attachments to professional healthcare provision highlighted the belief held by clinicians that patients desire the security, support and safely of in-centre care.

\section{An unrelenting imposition}

Almost all clinicians were convinced that many patients would be resistant to home haemodialysis and averse to bringing the disease home. Clinicians believed patients did not want dialysis to disrupt their normality and impinge on everyday lives. They predicted that patients might not wish to have a haemodialysis machine at home as it would serve as a constant reminder of their disease. Clinicians spoke of home haemodialysis as being confronting for family and friends and that patients would be unwilling to impose an unnecessary burden on their families.

\section{Carer burden}

Some clinicians were apprehensive that home haemodialysis would be an overwhelming burden on family carers. They believed that family members were not able perform dialysis, monitor the patient's health status, and manage complications. Clinicians felt that carers might experience considerable pressure, stress and anxiety.

\section{Attachments to professional healthcare provision}

Clinicians believed that patients relied on doctors for health-related issues and felt safer receiving care in a medical unit. At a community level, health education and promotion of self-management for chronic diseases was generally lacking in the participating regions. Many clinicians anticipated that patients would feel apprehensive and refuse to 'cut the umbilical cord with the clinic'. They perceived that home-based care might disconnect patients from social support of staff and other patients, impair access to clinicians and reduce safety. Clinicians felt that separation from in-centre care would be particularly difficult for patients with a longer previous experience of in-centre haemodialysis care. They believed that patients would be anxious about taking responsibility for complicated and technical medical tasks.

\section{Limited awareness}

The participants acknowledged that treatment choices made by patients are shaped by the way doctors present treatment options, which was almost always an 'active promotion' of in-centre haemodialysis. They believed that patients generally trust and willingly accept treatment recommendations without hesitation. Home haemodialysis was rarely discussed in clinic consultations and many patients were not aware that home haemodialysis might be a treatment choice.

\section{DISCUSSION}

The four themes encompassing clinician beliefs and perceptions of home haemodialysis in regions without established services (external structural barriers, centre capacity, clinician responsibility and motivation, and cultural apprehension) are useful when considering strategies to increase acceptance and uptake of home haemodialysis. Clinicians generally recognise the benefits of home haemodialysis for patients including increased empowerment, autonomy and flexibility; however, they are apprehensive about providing home haemodialysis services and doubt the feasibility of such programmes within their regions. Clinicians perceive that patient proximity to dialysis centres and readily available alternative dialysis modalities negate patient demand for home haemodialysis. Barriers to the establishment of home haemodialysis services included reimbursement rates for in-centre haemodialysis, cultural expectations of the clinician's role in medical care, as well as a strong perception that home haemodialysis could be an unrelenting, overwhelming and unwarranted burden on their families. Clinicians suggested that their own direct experience of home haemodialysis could provide confidence in home haemodialysis which, combined with their own existing professional interest in providing a broad range of treatment options to patients and families, would increase their motivation to advocate for home haemodialysis services.

Research on treatment choices when approaching the need for dialysis suggests that when patients are fully informed about dialysis modality options, about half choose self-care dialysis (peritoneal dialysis or 
haemodialysis) and another $15 \%$ choose limited-care clinic-based haemodialysis. ${ }^{10}$ Evidence also indicates that what a clinician believes about patient survival, treatment effectiveness and quality of life for each dialysis modality strongly influences the uptake of home haemodialysis. Conversely, limited training or experience in home therapies reduces clinician confidence in home-based care. ${ }^{13}$ A dialysis centre 'champion' for home haemodialysis is considered pivotal to increasing and sustaining homebased dialysis services. ${ }^{13}$ These data suggest that increasing clinician's knowledge and experience of home haemodialysis in areas without home dialysis services could facilitate the development of home haemodialysis and, in doing so, broaden dialysis treatment choices for patients.

Increased patient responsibility and control over the management of chronic diseases reduces symptom severity and improves both patient confidence and clinical outcomes. $^{27-29}$ Although home haemodialysis fulfils many tenets of patient-centred care ideology 27 and, accordingly, has the potential to improve outcomes for people who have end-stage kidney disease, it is apparent that shifting the responsibility for chronic illness management of end-stage kidney disease towards patients and carers is confronting for clinicians who are unfamiliar with and inexperienced in home haemodialysis. This perception is potentially in conflict with the views expressed by patients and carers with experience of earlier stages of chronic kidney disease not requiring dialysis for whom treatment choices are prioritised by maximising the quality of life. ${ }^{15}$

Financial reimbursement for home haemodialysis is a central concern for the interviewed clinicians. Central reimbursement and regulations governing funding for long-term dialysis vary considerably across healthcare jurisdictions and may be responsible in part for variable implementation of home haemodialysis services. Although home haemodialysis is markedly cheaper than in-centre care, ${ }^{31-33}$ few health systems differentiate between dialysis modalities in funding models. ${ }^{34}$ In The Netherlands and some states in Australia, home haemodialysis is incentivised with a higher reimbursement rate than for in-centre haemodialysis, and in the UK and Ontario, Canada, reimbursement of home haemodialysis is similar to in-centre care but reimbursement includes funding for longer hours or extended frequency dialysis at home. The Belgian health system provides a bonus if a certain number of patients in a facility perform self-care or home haemodialysis, peritoneal dialysis or transplantation, ${ }^{34}$ while in New Zealand, regional authorities are given overall budgets for renal services, effectively encouraging home therapies through the incentive for providers to lower overall costs of care. ${ }^{35}$ Funding models that incentivise home haemodialysis in areas of low uptake would similarly reduce costs to health systems as a whole and directly motivate clinicians and providers to prioritise new home dialysis programmes.

Our study included only clinicians within a large dialysis service provider in Europe and South America. While the study included a broad range of perspectives, the findings may not be generalisable to other countries or health provider settings. However, the findings resonate with barriers to home haemodialysis identified by other studies. $^{22} 36$ In common with other qualitative studies, we cannot assume that our findings are representative of the whole clinician population, although we actively sought a broad range of views and experiences through purposive sampling. We did not seek participant feedback on the transcripts or preliminary findings given the challenges of the different languages included. However, we used investigator triangulation where AT/MR debriefed after each interview, and reviewed the transcripts independently to ensure the thematic analysis encompassed the full range of participants' perspectives. For interviews with non-English participants, some cultural or linguistic nuances may not have been captured; however, the concepts and themes we identified were comparable with those that arose from interviews with English-speaking participants.

Inequalities in global access to home haemodialysis exist within many health systems. A better understanding of the barriers to home haemodialysis that are relevant to specific health systems and regions is critical to overcoming them. Using our thematic framework together with published international experience ${ }^{36-40}$ we suggest strategies to foster uptake of home haemodialysis (table 4). Clinician enthusiasm for a dialysis modality is a key determinant of the proportion of patients that are treated with that modality ${ }^{11}$; as such, addressing clinician attitudinal, knowledge and experience barriers may increase acceptance and motivation to establish home haemodialysis programmes. We suggest that increasing clinician competence and familiarity with home haemodialysis is a necessary component of expanding home haemodialysis care in regions without existing services. Professional education and training for clinicians could involve interactive seminars with healthcare providers who have experience in home haemodialysis, visits to other centres to gain hands-on clinical experience in home haemodialysis, ${ }^{36}$ and the support of a 'local champion' for home haemodialysis. ${ }^{11}$ Clinicians frequently voice concerns about patient safety suggesting that specific strategies are needed to minimise potential risks for clinicians and patients in home haemodialysis programmes. These might include $24 \mathrm{~h}$ access to a home haemodialysis clinician, increased patient and physician education and training, and specific carer support. More work is needed to determine fully patients' and clinicians' views on the design and delivery of such services.

The observational nature of the existing evidence for home haemodialysis together with the concerns that clinicians raise over the safety and durability of home haemodialysis support the need for a randomised trial evaluating the effectiveness and safety of home haemodialysis. In addition, research could evaluate the effectiveness of targeted interventions or strategies to overcome 
Table 4 Suggestions for overcoming clinician barriers to providing home haemodialysis

\section{Barriers \\ Lack of awareness and} experience in home haemodialysis

Concern about patient safety, adequacy of support, and psychosocial burden

\section{Potential solutions}

- Convene regional and national professional education seminars on home haemodialysis

- Incorporate home haemodialysis training in nephrology training programmes

- Develop and disseminate clinical practice guidelines on home haemodialysis

- Organise short-term visits to other centres to gain practical experience

- Identify local champions (professional advocates) for home haemodialysis

- Disseminate home haemodialysis 'success stories' through meetings, newsletters, nephrology society communiqués

- Establish centralised home haemodialysis training units to conserve resources and attract training staff

- Facilitate collaboration and interaction with professionals who have extensive experience with home haemodialysis

- Develop policies addressing patient safety including 24-h availability of technical and medical support; patient and carer training, individualised patient assessment for home visits or paid carers; patient access to a 'parent dialysis centre' in case of complications; regular patient contact; dedicated psychologist/social worker

- Ensure laboratory results can be tracked easily by patients and providers

- Coordinate independent accreditation to ensure quality of equipment and dialysis solutions; and conducive home environment

- Increase knowledge about the potential clinical benefits of home haemodialysis (use data of current practice to establish an evidence base to support research, which will reflect efficacy of outcomes of home programmes; and encourage participation in RCTs of home dialysis versus in-centre dialysis to strengthen evidence base for home HD)

- Educate clinicians about the availability of current 'patient-friendly' home haemodialysis machines (smaller size, minimise need for a family carer to assist)

- Demonstrate patient ability cope with home haemodialysis (self-cannulation, operating simple machines)

- Emphasise the importance of fostering patient independence and self-care rather than a 'learned helplessness'

- Provide respite opportunities for home HD patients to avoid patient and/or carer 'burn-out'

- Promote further development of simplified home HD machines that are portable and don't require significant plumbing or electrical changes to home

Limited centre capacity in dialysis centres to

- Allocate resources and dedicated space for training

- Provide home HD training facilities that are geographically separate from in-centre HD facilities establish home haemodialysis programmes Inadequate compensation and financial disadvantage

Competing centre priorities
- Emphasise 'patient-centred' care within the organisational culture to minimise influence of commercial interests (eg, to incorporate patient-orientated key performance indicators)

- Implement centralisation of funding away from commercial interests and reduce physician reimbursement on a fee-for-service model

- Compensate clinicians for 'hidden tasks' including the planning and management of home haemodialysis programmes

- Provide additional financial incentives to units including reimbursement at a higher than cost level

- Develop public sector funding models that rewards home haemodialysis programmes (eg, provide incentive payments for home haemodialysis patients

- Defray patients' out of pocket expenses for home HD (water, electricity)

- Highlight the importance of equity of access to all dialysis modalities (eg, patients may prefer home haemodialysis to in-centre haemodialysis)

- Provide balanced patient education early in pre-dialysis phase emphasising all dialysis modalities available such that patients are allowed to make an informed choice. the identified barriers to home haemodialysis (such as small-scale implementation of home haemodialysis in selected centres, patient-friendly technology, improved reimbursement and clinician education programmes) to increase the uptake of home haemodialysis.
This analysis suggests potential barriers to the development of home haemodialysis programmes specific to regions without active home haemodialysis services that include structural barriers, competing treatment choices and services, the role of the clinician in care and 
cultural perspectives within health delivery. Specific strategies to address these issues may increase access to home haemodialysis in some global regions.

Author affiliations

${ }^{1}$ The Centre for Kidney Research, The Children's Hospital at Westmead, Sydney, New South Wales, Australia

${ }^{2}$ Sydney School of Public Health, The University of Sydney, Sydney, New South Wales, Australia

${ }^{3}$ Department of Medicine, University of Otago Christchurch, Christchurch, New Zealand

${ }^{4}$ Department of Medicine and Community Health Sciences, University of Calgary, Calgary, Canada

${ }^{5}$ Diaverum Scientific Office, Medical-Scientific Office, Diaverum Renal Services Group, Lund, Sweden

${ }^{6}$ School of Medicine, University of Queensland at Princess Alexandra Hospital, Brisbane, Queensland, Australia

${ }^{7}$ Diaverum Medical Office, Diaverum Renal Services Group, Lund, Sweden

${ }^{8}$ Diaverum Renal Services Group, Lund, Sweden

${ }^{9}$ School of Medicine, Hofstra Northshore LIJ, Great Neck, New York, USA

${ }^{10}$ Department of Clinical Pharmacology and Epidemiology, Consorzio Mario

Negri Sud, S. Maria Imbaro, Italy

${ }^{11}$ Diaverum Scientific Office, Lund, Sweden

${ }^{12}$ Department of Emergency and Organ Transplantation, University of Bari, Bari, Italy

Acknowledgements We would like to thank all the clinicians who volunteered their time to participate in this study.

Contributors All authors, external and internal, had full access to all of the data in the study and can take responsibility for the integrity of the data and the accuracy of the data analysis. AT participated in the design of the study, did the interviews, transcribed the interviews, carried out the thematic analysis and drafted the manuscript. SP designed the study, participated in the thematic analysis and critical review of the first and subsequent manuscript drafts. BM and JCC provided intellectual input into first and subsequent manuscript drafts and assisted with thematic analysis. MR, LG, JH and MO assisted with interviews and provided intellectual input into subsequent manuscript drafts. DWJ assisted with thematic analysis and provided intellectual input into primary and subsequent drafts. JH and MO provided intellectual input into subsequent manuscript drafts. GFMS conceived the study, obtained funding, assisted with study design and thematic analysis, provided intellectual input into subsequent manuscript drafts and is the guarantor. All authors made substantial contributions to conception and design, acquisition of data or analysis and interpretation of data; drafting the article or revising it critically for important intellectual content; and provided final approval of the version to be published.

Funding The study has been part-funded by Diaverum AB. AT is supported by the National Health and Medical Research Council Early Career Fellowship ID 1037162; SP is supported in part by an by an unrestricted grant from Amgen Dompé administered by the Mario Negri Sud Consortium, Italy; DJ has received research grants, travel sponsorships and consultancy fees from Baxter Healthcare and Fresenius Medical Care and is a current recipient of a Queensland Government Health Research Fellowship. JH is Chief Medical Officer at Diaverum AB, a global provider of renal services; GFMS is Senior Vice President scientific affairs at Diaverum $A B$, a global provider of renal services.

Competing interests None.

Ethics approval Ethics approval was obtained for the study as follows: for participants from France we received ethic approval from Comitè de Protection des Personnes Sud-Mediterranèe II, 2011-A01044-37; for participants from Diaverum (Portugal, Sweden, Germany, Argentina) we received ethic approval from Comissão de Etica da Diaverum, aprovacão n. 01/2011; for Italy we informed ethics committees according to Italian law (Italian Republic. Determination of the Italian Medicines Agency of 20 March 2008 [in Italian]. Official Gazette of the Italian Republic. General Series No. 76; March 31, 2008); for Argentina there was no requirement for ethics submissions for this type of study. All participants provided written voluntary informed consent.

Provenance and peer review Not commissioned; externally peer reviewed.

Data sharing statement No additional data are available.

\section{REFERENCES}

1. Atkins RC. The changing patterns of chronic kidney disease: the need to develop strategies for prevention relevant to different regions and countries. Kidney Int 2005;68(S98):S83-5.

2. Renal disease in minority populations and developing nations. Proceedings of a satellite meeting of the World Congress of Nephrology; April 25-27, 2007. Petropolis, Brazil. Ethnicity disease 2009;19(1 Suppl 1):S1-1-89.

3. Cass A, Chadban S, Gallagher M, et al. The economic impact of end-stage kidney disease in Australia. Projections to 2020. http://www.kidney.org.au (accessed 1 March 2012): Kidney Heath Australia, Melbourne, Australia, 2010.

4. Julius $M$, Kneisley JD, Carpentier-Alting $P$, et al. A comparison of employment rates of patients treated with continuous ambulatory peritoneal dialysis vs in-center hemodialysis (Michigan End-Stage Renal Disease Study). Arch Int Med 1989;149:839-42.

5. Pauly RP, Gill JS, Rose CL, et al. Survival among nocturnal home haemodialysis patients compared to kidney transplant recipients. Nephrol Dial Transpl 2009;24:2915-9.

6. Rocco MV, Lockridge J, Beck GJ, et al. The effects of frequent nocturnal home hemodialysis: the Frequent Hemodialysis Network Nocturnal Trial. Kidney Int 2011;80:1080-91.

7. MacGregor MS, Agar JW, Blagg CR. Home haemodialysis-international trends and variation. Nephrol Dial Transpl 2006;21:1934-45.

8. Honkanen EO, Rauta VM. What happened in Finland to increase home hemodialysis? Hemodial Int 2008;12(Suppl 1):S11-15.

9. George CR. Caring for kidneys in the antipodes: how Australia and New Zealand have addressed the challenge of end-stage renal failure. Am J Kidney Dis 2009;53:536-45.

10. Goovaerts T, Jadoul M, Goffin E. Influence of a pre-dialysis education programme (PDEP) on the mode of renal replacement therapy. Nephrol Dial Transpl 2005;20:1842-7.

11. Castledine C, Gilg J, Rogers C, et al. UK Renal Registry. The thirteenth annual report. Bristol, UK: The Renal Association, 2010.

12. Damschroder LJ, Aron DC, Keith RE, et al. Fostering implementation of health services research findings into practice: a consolidated framework for advancing implementation science. Implement Sci 2009;4:50.

13. Casteldine C, Gilg J, Rogers C, et al. RRT incidence and use of home dialysis modalities. The UK Renal Registry 13th Annual Report. Chapter 15. 2010:263-75.

14. Agar JW, Knight RJ, Simmonds RE, et al. Nocturnal haemodialysis: an Australian cost comparison with conventional satellite haemodialysis. Nephrology (Carlton, Victoria) 2005;10:557-70.

15. Morton RM, Tong A, Howard $\mathrm{K}$, et al. The views of patients and carers in treatment decision making for chronic kidney disease: systematic review and thematic synthesis of qualitative studies. BMJ 2011;340:c112.

16. Morton RM, Tong A, Webster AC, et al. Characteristics of dialysis important to patients and family caregivers: a mixed methods approach. Nephrol Dial Transp/ 2011:Online first:doi:10.1093/ndt/gfr177 2011;26:4038-4046.

17. Derrett S, Darmody M, Williams S, et al. Older peoples' satisfaction with home-based dialysis. Nephrology 2010;15:464-70.

18. Fadem SZ, Walker DR, Abbott G, et al. Satisfaction with renal replacement therapy and education: the American Association of Kidney Patients survey. Clin J Am Soc Nephrol 2011;6:605-12.

19. Qamar M, Bender F, Rault R, et al. The United States' perspectives on home dialysis. Adv Chronic Kidney Dis 2009;16:189-97.

20. Manns B, Johnson JA, Taub K, et al. Quality of life in patients treated with hemodialysis or peritoneal dialysis: what are the important determinants. Clin Nephrol 2003;60:341-51.

21. Lee A, Gudex C, Povlsen JV, et al. Patients' views regarding choice of dialysis modality. Nephrol Dial Transpltion 2008;23:3953-9.

22. Cafazzo JA, Leonard K, Easty AC, et al. Patient-perceived barriers to the adoption of nocturnal home hemodialysis. Clin J Am Soc Nephrol 2009;4:784-9.

23. Cases A, Dempster M, Davies M, et al. The experience of individuals with renal failure participating in home haemodialysis: an interpretative phenomenological analysis. J Health Psychol 2011;16:884-94.

24. Diaz-Buxo JA, Crawford-Bonadio TL, St Pierre D, et al. Establishing a successful home dialysis program. Blood Purif 2006;24:22-7. 
25. Liamputtong P. Qualitative research methods. Melbourne: Oxford University Press, 2009.

26. Kvale S. Interviews. Thousand Oaks, CA: Sage Publications, 1996.

27. Department of Health. The expert patient: a new approach to chronic disease management for the 21st century. London, UK: Stationery Office: Department of Health, 2001.

28. Barlow J, Wright C, Sheasby J, et al. Self-management approaches for people with chronic conditions: a review. Patient Educ Counsel 2002;48:177-87.

29. Lorg K, Sobel DS, Steward AL, et al. Evidence suggesting that a chronic disease self-management program can improve health status while reducing hospitalization: a randomized trial. Med Care 1999;37:5-14.

30. Gerteis M. Through the patient's eyes: Understanding and promoting patient-centered care. San Francisco: Jossey-Bass Publishers, 1993.

31. Winkelmayer WC, Weinstein MC, Mittleman MA, et al. Health economic evaluations: the special case of end-stage renal disease treatment. Med Decis Making 2002;22:417-30.

32. Goeree R, Manalich J, Grootendorst P, et al. Cost analysis of dialysis treatments for end-stage renal disease (ESRD). Clin Invest Med 1995;18:455-64.

33. Croxson BE, Ashton T. A cost effectiveness analysis of the treatment of end stage renal failure. N Z Med J 1990;103:171-4.
34. Vanholder R, Davenport A, Hannedouche T, et al. Reimbursement of dialysis: a comparison of seven countries. J Am Soc Nephrol 2012;23:1291-8.

35. Dor A, Pauly MV, Eichleay MA, et al. End-stage renal disease and economic incentives: the International Study of Health Care Organization and Financing (ISHCOF). Int $J$ Health Care Finance Econ 2007;7:73-111.

36. Golper TA, Saxena AB, Piraino B, et al. Systematic barriers to the effective delivery of home dialysis in the United States: a report from the public policy/advocacy committee of the North American Chapter of the International Society for Peritoneal Dialysis. Am J Kidney Dis 2011:58:879-85.

37. Agar JWM, Hawley CM, Kerr PG. Home hemodialysis in Australia and New Zealand: how and why it has been successfull. Sem Dial 2011:24:658-63.

38. Ludlow MJ, George CRP, Hawley C, et al. How Australian nephrolgists view home dialysis: results of a national survey. Nephrology 2011;16:446-52.

39. Blagg CR. Home haemodialysis: 'home, home, sweet, sweet home!'. Nephrology 2005;10:206-14.

40. Lynn KL, Buttimore AL. Future of home haemodialysis in Australia and New Zealand. Nephrology 2005;10:231-3. 